\title{
Hadronic properties of the photon
}

\author{
Sergey Gevorkyan ${ }^{1, *}$ \\ ${ }^{1}$ LHEP, Joint Institute for Nuclear Research, Dubna, Russia
}

\begin{abstract}
Currently it is no doubt that high energy photons (real or virtual) have a hadronic component leading to photon shadowing in its interaction with nuclei. We shortly consider the difficulties appeared in the models like vector dominance model (VDM) and stress that these problems can be solved in a color dipole model inspired by Quantum Chromodynamics (QCD).

From the other hand, the color dipole model allows one to investigate the impact of vector meson polarization on their interaction with nucleons and nuclei, the challenge which is crucial for studying, for instance, such a fundamental effect as color transparency.
\end{abstract}

\section{Introduction}

At first the photon was regarded as structureless, but with increasing energy it was found that through the interaction with a Coulomb field of a target the photon could materialize as pairs of electrons, the effect which first manifested on the photon structure. The energy threshold depends on the target. For instance, the pair production begins at $E_{\gamma}=2 m_{e}$ in the Coulomb field of a nucleus and at $E_{\gamma}=4 m_{e}$ in the photoproduction of the atomic electron. ${ }^{1}$

At higher energy $E_{\gamma}>2 m_{\pi}$ the hadronic structure of the photon is clearly manifested. Before the interaction the photon (real or virtual) fluctuates to the hadronic state, which then interacts with a target. A good example of this two step interaction is the VDM, where the photon hadronic component is presented as a set of light vector mesons $\rho, \omega, \phi$ etc. The lifetime of this fluctuation or coherence length grows with energy as $\tau_{c}=l_{c}=\frac{2 E}{M^{2}+Q^{2}}$, where $M$ and $Q$ are the masses of the hadronic state and virtual photon, correspondingly.

The best way to reveal the existence of the hadronic component in photon is its interaction with nuclei. If the hadronic component lifetime is long enough it leads to absorption of photons (shadowing) in their interaction with nuclei [1]. The imaginary part of the forward $\gamma A \rightarrow \gamma A$ amplitude defining the total photon-nucleus cross section can be represented as a sum of two terms: elastic photon scattering on the nucleon in the nucleus and two step photon scattering via production of some hadronic state $\mathrm{X}$ with its subsequent conversion to the photon (Fig. 1).

At energies when the coherence length becomes much larger than nucleus dimension $l_{c}>R$, the picture looks as if the pure hadronic state $\mathrm{X}$ interacts with a nucleus leading to absorption and relevant changes in the atomic mass dependence (A-dependence). As it is

\footnotetext{
*e-mail: gevs@jinr.ru

${ }^{1}$ Lepton pair production $\gamma \leftrightarrows e^{+} e^{-}$and vice versa at any energies is accounted by taking into account radiative corrections in Quantum Electrodynamic
} 


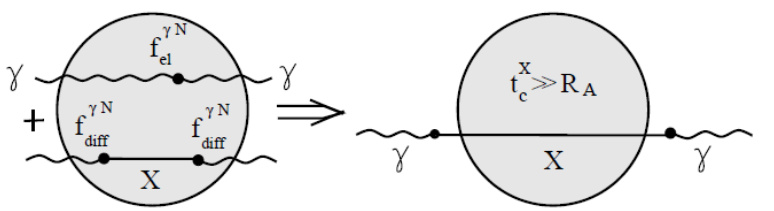

Figure 1. Left: direct and two step contribution to photon forward scattering. Right: limit of high energies

seen from Figs. 2 and 3 the shadowing of photon (real or virtual) in nuclei really takes place, but the photon absorption is weaker than theoretical predictions.

The similar transition has been predicted [2] for any meson incoherent photoproduction off nucleus. In this case besides the usual absorption of mesons produced in direct interactions of photon with nucleon in nucleus the photon can produce a vector meson, which after multiple scattering in nucleus gives the final meson meson $M$. The interference of the direct and two step contributions leads to decreasing the nuclear transparency $A_{e f f}=\frac{d \sigma\left(\gamma A \rightarrow M A^{\prime}\right)}{d \sigma(\gamma N \rightarrow M N)}$ with increasing of the photon energy or, in other words, with the coherence length increase.

It can be seen from the right picture in Figs. 2 and 3 that this decrease with energy is visible in $\pi^{0}$ photoproduction on different nuclei [3], but it is much slower than theoretical predictions.
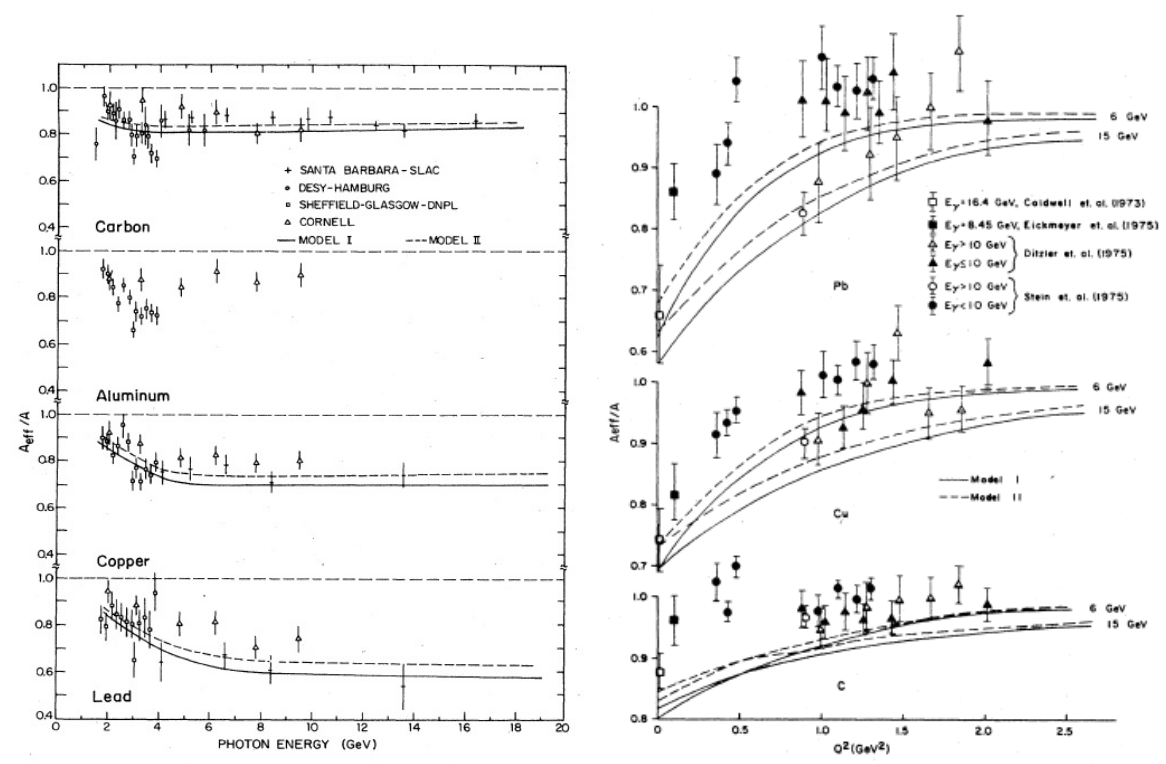

Figure 2. Energy and $Q^{2}$ dependencies of $A_{e f f}=\frac{\sigma(\gamma A)}{A \sigma(\gamma N)}$ for real and virtual photons

At present it is no doubt that the photon has a hadronic component leading to photon shadowing in the nucleus. The models as the vector dominance model predict more shadowing and stronger suppression of nuclear transparency than it is shown in existing experimental data. From our point of view this discrepancy is the result of tacit assumption of equality of elementary amplitudes of production and absorption in a two step term. This assumption cannot be valid since the finite coherence length should lead to the difference between these amplitudes which grows with energy. The challenge to solve this problem gives the color dipole 


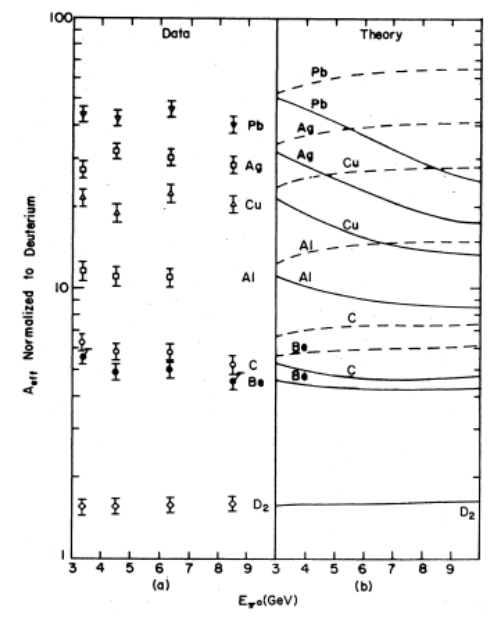

Figure 3. The energy dependence of $A_{\text {eff }}$ in $\pi^{0}$ photoproduction on different nuclei

model first applied to photoproduction [4] and electroproduction [5] on nucleon, which we shortly consider.

\section{Color dipole model}

Currently the best way to describe the hadronic photon structure is the color dipole model widely discussed in literature. In any QCD description of photon interactions with the target the first step is the conversion of the initial photon into a quark-antiquark pair, which is then followed by its interaction with the target (Fig. 4). This colorless quark-anti-quark pair interacts with a target as a color dipole and its cross section depends only on the separation $r$ in the pair and due to gauge invariance $\sigma(r) \sim r^{2} \log (r)$ at $r \rightarrow 0$. Generalization of this approach to photon nucleus interactions [6] allows one to correctly account, besides elastic (Glauber) multiple scattering, all inelastic (Gribov) corrections and reconcile the theoretical predictions with experimental data. Unlike the VDM this QCD inspired approach explained by the scaling behavior of the structure function in electroproduction and allows one to investigate separately the interaction of transverse and longitudinal virtual photons and vector mesons with the target. The expressions in the model are transparent and well motivated. In this model the total cross section of the transverse $T$ and longitudinal $L$ photons interaction with a target reads:

$$
\sigma_{L(T)}(\gamma A)=\int\left|\Psi_{L(T)}^{\gamma}\left(Q^{2}, r, \alpha\right)\right|^{2} \sigma(r) d^{2} r d \alpha
$$

where $r \equiv x_{\perp}$ - the transverse distance in $q \bar{q}$ pair, $\alpha$ - the partition of the light cone momentum carried by the quark, $(1-\alpha)$-anti-quark.

$\Psi_{L(T)}^{\gamma}\left(Q^{2}, r, \alpha\right)$ is the quark distribution in the transverse and longitudinal photon. The convenient parametrization of the dipole cross section $\sigma(r)=\sigma_{0}\left(1-e^{-\frac{r^{2}}{R^{2}}}\right)$ allows one to give the theoretical predictions not only for photoproduction, but also for production and scattering by hadron beams.

\section{The impact of vector meson polarization on its interaction with matter}

The vector mesons $V(\rho, \omega, \varphi)$ etc. can be transversely ( helicity $\lambda= \pm 1$ ) or longitudinally $(\lambda=0)$ polarized. Changing in Eq. (1) the photon distribution by the vector meson wave 


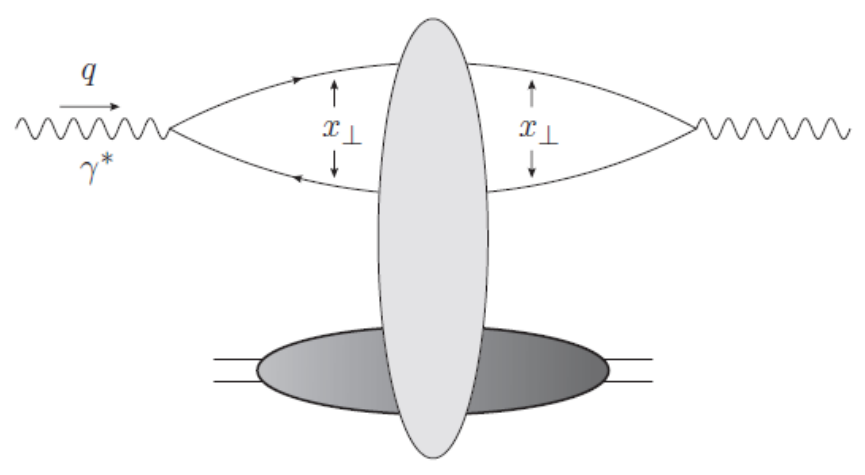

Figure 4. The virtual photon interaction with a target in a color dipole model

function it is possible to calculate the total cross sections of the transverse and longitudinally polarized vector meson with nucleon. As it is discussed in [7] they appear very different in realistic models. The knowledge of $\sigma_{T}(V N)$ and $\sigma_{L}(V N)$ is important since their difference leads to different absorption in nuclei depending on their polarization and has to be accounted, for instance, in studing the effect of color transparency.

According to QCD hard exclusive processes select configurations, where the quarks are close together forming a color neutral object with a transverse size $r \sim 1 / Q$. This effect is well known in QED in the photoproduction of $e^{+} e^{-}$pairs (Chudakov effect). The effect of color transparency is seen in electroproduction of vector mesons off nuclei as growth of nuclear transparency $T_{A}=\frac{d \sigma_{A}}{A d \sigma_{N}}$ with the mass of virtual photon $Q^{2}$. From the other hand, this growth can be in part due to the growth in electroproduction the relative contribution of longitudinally polarized vector meson, which has a less absorption in the matter than the transverse one. In Fig. 5 we cite the $Q^{2}$ dependence of $A^{e f f}$ for $\rho$ meson electroproduction off C and Fe nuclei. The curve denoted by stars is a result of our calculations of $A_{e f f}$ accounting for different absorption of $\rho$ meson in nuclei depending on its polarization. For the dependence of the spin density matrix element $\rho_{00}$ on $Q^{2}$ we have used the well known relation $\rho_{00}=\frac{\epsilon R}{(1+\epsilon R)}$ and the fit [8] for the ratio of the longitudinal to transverse cross section: $R\left(Q^{2}\right)=c_{0}\left(\frac{Q^{2}}{m^{2}}\right)^{c_{1}}$ with constants $\epsilon=0.8, c_{0}=0.56, c_{1}=0.47$.

As it is seen from Fig. 5 the considered effect has to be separated from the effect of color transparency as it is not small and exhibits the same behavior. Thus, the impact of vector meson polarization on its interaction with nucleus and nucleon can be crucial to determine such fundamental effect as color transparency.

\section{Summary}

The color dipole model inspired by QCD is a powerful tool to investigate the interaction of high energy photons (real or virtual) with nucleons and nuclei. This approach allows one to solve the long standing discrepancies between theoretical predictions and experimental data and study the impact of vector meson polarization on strong interactions.

There is a new proposal at CERN [9] to measure vector meson production off nuclei by the pion and kaon beams to extract their transverse and longitudinal interaction cross sections 

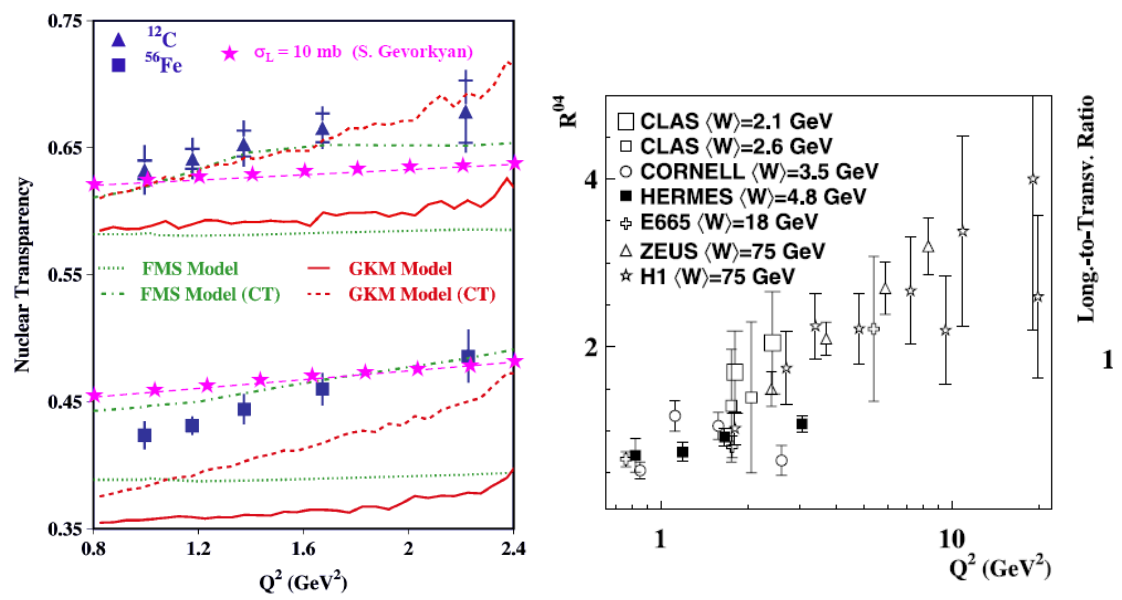

Figure 5. Left: Nuclear transparency $A_{e f f}$ as a function of photon virtuality $Q^{2}$. Right: The ratio $R=\frac{\sigma\left(\gamma^{*}+N \rightarrow V_{L}+N\right)}{\sigma\left(\gamma^{*}+N \rightarrow V_{T}+N\right)}$ as a function of $Q^{2}$

with nucleon as a result of different absorption. The deal is that in charge exchange reactions like $\pi^{-}+N \rightarrow V+N, \quad K^{-}+N \rightarrow V+N$ many longitudinally polarized vector mesons can be produced, i.e. charge exchange processes on different nuclei are the ideal laboratory to extract the longitudinal vector meson nucleon cross section $\sigma_{L}(V N)$.

\section{References}

[1] T. Bauer et al., Rev. of Modern Phys. 50, 261 (1978)

[2] K. Gottfried, D. Yennie, Phys. Rev. 182, 1595 (1969)

[3] H. Meyer at al., Phys. Rev. Lett. 28, 1344 (1972)

[4] S. Gevorkyan, A. Kotzinian, V. Jaloyan, Phys. Lett. B 212, 251 (1988)

[5] N. Nikolaev, B. Zakharov, Zeitshrift fur Phys. C 53, 331 (1992)

[6] B. Kopeliovich, J. Raufeisen, A. Tarasov, Phys. Lett. B 440, 151 (1998)

[7] S. Gevorkyan, EPJ WEB of Conference 138, 08004 (2017). JPCS 678, 012033 (2016)

[8] A. Airapetian et al., Eur. Phys. J. C 62, 659 (2009)

[9] B. Adams et al., Letter of Intend. A New QCD facility at the M2 beam line of the CERN SPS, arXiv:1808.00848 [hep-ex] 